\title{
Opinión de los estudiantes de odontología de la FES Iztacala UNAM, sobre el proceso de evaluación a distancia antes de la pandemia por COVID-19: estudio transversal.
}

\author{
Opinion of dental students at FES Iztacala UNAM \\ on the distance evaluation process before the \\ COVID-19 pandemic: a cross-sectional study.
}

Ricardo A Rivas Muñoz, * Eduardo Ensaldo Fuentes, * Eduardo Ensaldo Carrasco ${ }^{\ddagger}$

\section{RESUMEN}

Introducción: El proceso de evaluación a distancia es una modalidad que ha sido utilizada en los últimos años. Sin embargo, la pandemia por COVID-19 ha ocasionado su rápida adaptación. El personal docente ha reportado las ventajas de este modo de evaluación; no obstante, la opinión de los estudiantes de odontología en torno a las ventajas y desventajas de esta modalidad no ha sido explorada. Objetivo: Explorar las ventajas y desventajas de las tecnologías de la información y comunicación (TIC), específicamente en el proceso de evaluación a distancia reportadas por estudiantes de odontología. Material y métodos: Se realizó un estudio transversal retrospectivo, en una muestra no aleatoria de 310 estudiantes de tercer año, distribuidos en cuatro generaciones, adscritos a la Facultad de Odontología de la FES Iztacala. Se administró un cuestionario estructurado para la medición de sus opiniones en torno a las evaluaciones a distancia. Se realizó un análisis descriptivo y bivariado. Resultados: De un total de los 382 estudiantes potencialmente elegibles, 310 aceptaron participar (96\%). Conclusiones: La opinión mayoritaria fue la preferencia a los exámenes por Internet y mejor a distancia. Queda pendiente la duda: ¿cuál será el comportamiento cuando los estudios profesionales vuelvan a la normalidad después de la pandemia COVID-19?

Palabras clave: COVID-19, evaluación, enseñanza mixta, opinión estudiantil.

\section{ABSTRACT}

Introduction: The process of distance assessment has been employed in the last years. However, COVID-19 pandemic has accelerated its adoption. Academic personnel have previously reported the benefits of this mode of assessments. Nevertheless, dentistry-students'opinions around the advantages and disadvantages of this mode of assessment has not been explored. Objective: Explore the advantages and disadvantages concerning the ICT skills specifically about the process of distance assessment of dental students. Material and methods: A retrospective cross-sectional study was conducted in a non-randomized sample of 310 of third-year students distributed in four years attending to the School of Dentistry at FES Iztacala. A structured questionnaire was distributed to measure students' opinions around the advantages and disadvantages of distance assessments. A descriptive and bivariate analysis was conducted. Results: Questionnaires were returned by 310 students (96\% response rate). Conclusion: Students' perceptions of their ICT skills has increased, matched by better equipment and greater appreciation of e-learning.

Keywords: COVID-19, assessment, blended learning, student opinion.

\footnotetext{
* Profesor adscrito a la Facultad de Estudios Superiores (FES) Iztacala, Universidad Nacional Autónoma de México (UNAM). México.

‡ Jefe del Departamento de Investigación, Comisión Nacional de Arbitraje Médico (CONAMED). México.

Recibido: 07 de diciembre de 2021. Aceptado: 11 de enero de 2022.
}

Citar como: Rivas MRA, Ensaldo FE, Ensaldo CE. Opinión de los estudiantes de odontología de la FES Iztacala UNAM, sobre el proceso de evaluación a distancia antes de la pandemia por COVID-19: estudio transversal. Rev ADM. 2022; 79 (1): 7-11. https://dx.doi.org/10.35366/103812 


\section{INTRODUCCIÓN}

L a Organización Mundial de la Salud (OMS) reconoce desde el 20 de enero de 2020 a COVID-19 como una emergencia de salud pública a nivel internacional. Hasta el 15 de noviembre de 2021 se han identificado en todo el mundo un total de 253'163,330 casos confirmados de COVID-19 y 5’098,174 muertes confirmadas. De los cuales, la región de las Américas ocupa el primer lugar con 95'120,017 casos confirmados. Desde entonces, los países miembros de la OMS han implementado medidas locales para minimizar el riesgo de propagación de COVID-19 en la comunidad. Éstas han consistido en la restricción de viajes a países y/o ciudades afectadas, apoyado por la limitación de actividades al aire libre, actividades escolares presenciales, así como la implementación de campañas de vacunación a gran escala.

Por otra parte, es indudable que la comunidad estudiantil y profesorado universitario han mejorado sus habilidades con respecto a las tecnologías de la información y comunicación (TIC), unido a equipamiento informático tanto institucional como individual, haciendo evidente la conveniencia del denominado B-learning, esto es, el aprendizaje mixto, la combinación de la educación a distancia con la presencial. ${ }^{1,2}$

A casi más de dos años de la pandemia, existe la necesidad de los países y sus habitantes de continuar con las actividades económicas y escolares. Apoyado por la lenta reincorporación de la sociedad a las actividades, el sector educativo ha adoptado estrategias de educación a distancia tanto para la impartición de clases como de su evaluación a través de exámenes administrados por Internet. ${ }^{3-5}$ En este contexto, la rápida adaptación de los programas académicos presenciales hacia un modelo, ya sea puramente en línea o mixto, representa un reto para programas de licenciatura en el área de la salud, al considerar que estos programas involucran actividades realizadas en clínicas de enseñanza en odontología. ${ }^{1,2}$ Sin embargo, los sistemas de evaluación de los estudiantes de manera remota han existido y se han implementado previo a la pandemia por COVID-19. Un ejemplo de ello es la plataforma Moodle, la cual forma parte del sistema de evaluación del Campus Universitario Virtual de Educación a Distancia (CUVED) y que fue desarrollado por el Laboratorio de Evaluación y Educación Digital (LEED) ubicado en la Facultad de Estudios Superiores Iztacala.

Durante cinco años lectivos (2014 a 2018), y previo a la pandemia por COVID-19, en la asignatura de Endodoncia en la Facultad de Odontología de la FES Iztacala, se han aplicado evaluaciones a distancia a través de la plataforma Moodle. En este contexto, y en contraste con las ventajas de las evaluaciones presenciales, la experiencia reportada por el personal docente hacia la evaluación a distancia refiere ventajas. No obstante, la opinión de los estudiantes de odontología en torno a las ventajas y desventajas de esta modalidad no ha sido explorada. Por lo tanto, el propósito de este estudio fue explorar las ventajas y desventajas del proceso de evaluación a distancia reportadas por los estudiantes de odontología.

iQué lejos estábamos, entonces, que un día no íbamos a poder elegir! La modalidad de los exámenes a distancia se hizo indispensable por culpa de la pandemia, todos tuvimos que aprender si no lo sabíamos, o en el mejor de los casos, si teníamos alguna experiencia, aplicar obligatoriamente las nuevas herramientas basadas en la informática. ${ }^{6,7}$

\section{Fundamentación teórica}

Autores como Morgan y $\mathrm{O}^{\prime}$ Reilly ${ }^{8}$ conciben «la evaluación como la maquinaria (motor) que dirige y da forma al aprendizaje, más que simplemente un evento final que califica y reporta el desempeño». Consideran que este enfoque permite encontrar nuevas oportunidades para promover a través de la evaluación tipos de aprendizaje más útiles y deseables. Para estos autores la «evaluación formativa comprende todas aquellas actividades diseñadas para motivar, para aumentar la comprensión y para proporcionar a los estudiantes una indicación de sus progresos». Destacan que «facilitar una evaluación formativa apropiada y efectiva es uno de los aspectos más importantes del rol de cualquier profesor, y es vital para la confianza y sentido de progreso del estudiante». Con respecto a la evaluación sumativa, expresan que su principal propósito «es registrar o reportar una estimación de los logros de los estudiantes». De manera frecuente, se realiza al final del curso y conduce a calificaciones. ${ }^{9}$ Estos autores ${ }^{10,11}$ proponen los siguientes aspectos clave relativos a las evaluaciones en diferentes modalidades a distancia:

Clara fundamentación y enfoque pedagógico consistente. Las decisiones que se tomen respecto a las evaluaciones a distancia deben tener bases fundamentales explícitas y no sólo la promesa de un aprendizaje centrado en el alumno. Asimismo, dado que las tareas evaluativas orientan el aprendizaje, deben ser significativas y desarrollar las habilidades deseadas. El enfoque pedagógico que se utilice debe ser consecuente y muy alineado con todos los componentes del proceso instruccional, y entre ellos las decisiones acerca de las evaluaciones. 
Valores, propósitos, criterios y estándares explícitos. Los valores que apuntalan el diseño de la evaluación, así como los criterios utilizados para juzgar el logro de los estudiantes deben ser conocidos por ellos. Esto puede ayudarles a tomar decisiones sobre la forma de enfocar su aprendizaje.

Tareas de evaluación auténticas y holísticas. Los estudiantes se motivan para ocuparse con eventos de la vida real, problemas de sus propios mundos y puestos de trabajo cuando se asignan tareas de evaluación auténticas. Las tareas holísticas crean oportunidades para que los estudiantes a distancia se comprometan con evaluaciones aplicadas, tales como los estudios de casos, escenarios y proyectos.

Grado facilitativo de estructura. Tiene como propósito facilitar intencionalmente y de manera progresiva en el alumno habilidades dirigidas al logro de la autodirección, tales como recuerdo de la información, establecimiento de objetivos, pensamiento crítico, autogestión y autoevaluación, promoviendo así el cambio del control del profesor al alumno.

Suficiente evaluación formativa y a tiempo. «La evaluación formativa y la sumativa deben entrelazarse estratégicamente para motivar y proporcionar alguna estructura al aprendizaje, crear una fuente de diálogo, y ayudar a que los alumnos obtengan una visión de su progreso.» ${ }^{12}$

Conocimiento del contexto de aprendizaje y percepciones. La planificación de las evaluaciones a distancia debe considerar el conocimiento de los contextos de los alumnos, así como sus percepciones acerca de las tareas de evaluación.

Existe una clara diferencia con las evaluaciones presenciales tradicionales que ni siquiera planean alcanzar estos objetivos, puesto que se vuelven repetitivos sin tomar en cuenta a los estudiantes ni sus necesidades. ${ }^{13}$

\section{MATERIAL Y MÉTODOS}

Se realizó un estudio transversal en una muestra no aleatoria por conveniencia de 310 estudiantes de odontología adscritos a la Facultad de Estudios Superiores (FES) Iztacala de la Universidad Nacional Autónoma de México (UNAM).

Instrumento de medición. Se diseñó un cuestionario de siete preguntas estructuradas y cinco preguntas abiertas. Seis de las preguntas estructuradas permitían una sola respuesta y una permitía respuestas múltiples. La encuesta fue anónima para facilitar la veracidad y se computó hasta que terminaron las cuatro generaciones investigadas. Se aplicaron 350 encuestas. El cuestionario conservó las mismas preguntas para todas las generaciones investigadas.

Análisis. Se realizó un análisis descriptivo de las características generales de los participantes. Asimismo, se realizó un análisis bivariado para identificar posibles diferencias significativas en la distribución de las respuestas en función del año de aplicación del cuestionario y sexo.

\section{RESULTADOS}

De un total de 382 estudiantes potencialmente elegibles, 310 aceptaron participar de manera voluntaria (tasa de respuesta de 96\%), de los cuales 223 (71.9\%) fueron mujeres y $87(28.1 \%)$ hombres. ${ }^{14}$

De acuerdo con los datos recabados, la gran mayoría de las evaluaciones que los estudiantes habían experimentado habían sido en la modalidad presencial, por escrito o algunas veces oralmente.

Los denominados «exámenes departamentales»y una asignatura habían empleado la modalidad «en línea» aprovechando los laboratorios de informática que ofrecía la propia FES Iztacala.

Sólo la asignatura de Endodoncia había aplicado la modalidad a distancia, respondiendo sus evaluaciones desde fuera de las instalaciones universitarias. El 86\% marcó preferencia por la evaluación a distancia sobre la evaluación presencial. ${ }^{15}$

\section{DISCUSIÓN}

Las metas de este cuestionario fueron obtener retroalimentación con las opiniones de los estudiantes de la carrera de Cirujano Dentista de la FES Iztacala, UNAM, basadas en sus capacidades en la TIC entre sus experiencias en la evaluación presencial y la evaluación a distancia o utilizando la informática instalada en la propia institución. La Tabla 1 muestra las principales ventajas y desventajas que, según la opinión de los alumnos, tienen tanto la evaluación presencial como a distancia, con el fin de que sea la comunidad académica quien decida cuál será su elección (cuando ésta vuelva a ser posible, ya que en este momento aún es incierta).

\section{CONCLUSIONES}

Los objetivos de este cuestionario fueron obtener retroalimentación en la aplicación de la evaluación a distancia, cuando todavía había la posibilidad de escoger la evaluación presencial tradicional, basados en la opinión de 
Tabla 1: Ventajas y desventajas de las dos modalidades experimentadas, según la opinión de los estudiantes.

\begin{tabular}{|c|c|}
\hline Evaluación presencial & Evaluación a distancia \\
\hline \multicolumn{2}{|c|}{ Ventajas } \\
\hline $\begin{array}{l}\text { En caso de dudas, se puede consultar al profesor } \\
\text { Son menos estresantes } \\
\text { No hay cronómetro } \\
\text { Son más frecuentes las respuestas abiertas } \\
\text { No es necesario equipo especial para responder }\end{array}$ & $\begin{array}{l}\text { No son necesarios los formatos } \\
\text { El horario es más flexible } \\
\text { Se pueden consultar apuntes } \\
\text { Se realizan con más tranquilidad } \\
\text { Es menos personal }\end{array}$ \\
\hline \multicolumn{2}{|c|}{ Desventajas } \\
\hline $\begin{array}{c}\text { Puede haber presión del supervisor } \\
\text { En ocasiones se auxilia a ciertos estudiantes } \\
\text { No hay cronómetro } \\
\text { Son más frecuentes las respuestas abiertas } \\
\text { Hay más distractores }\end{array}$ & $\begin{array}{c}\text { No se pueden aclarar dudas respecto a la redacción } \\
\text { Respuestas similares y confusas } \\
\text { Es más fácil hacer trampas } \\
\text { Problemas con conexión } \\
\text { No siempre se cuenta con el equipo requerido }\end{array}$ \\
\hline
\end{tabular}

los estudiantes que habían experimentado ambas modalidades. Los estudiantes investigados están en el tercero de cuatro años de su carrera profesional.

La circunstancia actual de la educación superior en carreras teórico-prácticas, como es la odontología, todavía es incierta, debido a que la pandemia COVID-19 no ha sido erradicada. Por lo que aún no es posible planear a mediano y largo plazo el mejor tipo de evaluación. Sin embargo, es necesario seguir con atención la opinión de los estudiantes para adecuar el mejor método cuando vuelva a ser posible elegir entre los sistemas presenciales, mixtos o a distancia. ${ }^{7}$

\section{REFERENCIAS}

1. Elsalem L, Al-Azzam N, Jum'ah AA, Obeidat N, Sindiani AM, Kheirallah KA. Stress and behavioral changes with remote E-exams during the Covid-19 pandemic: A cross-sectional study among undergraduates of medical sciences. Ann Med Surg (Lond). 2020; 60: 271-279.

2. Elsalem L, Al-Azzam N, Jum'ah AA, Obeidat N. Remote E-exams during Covid-19 pandemic: A cross-sectional study of students' preferences and academic dishonesty in faculties of medical sciences. Ann Med Surg (Lond). 2021; 62: 326-333.

3. Echagaray P, Marra A, Coronado M. Enseñar odontología en tiempos de pandemia: virtualidad, oportunidades y límites. Facultad de Odontología. Universidad Nacional de Cuyo. 2020; 14 (2): 11-16.

4. Guevara-Veliz DN, Flores-Joaquin KM, Maturrano-Santos AG, MattosVela MA. Educación virtual en odontología durante la pandemia por COVID-19. Rev Cient Odontol (Lima). 2021; 9 (3): e078.

5. Iurcov R, Pop LM, lorga M. Impact of COVID-19 pandemic on academic activity and health status among romanian medical dentistry students; a cross-sectional study. Int J Environ Res Public Health. 2021; 18 (11): 6041.

6. Cayo-Rojas CF, Agramonte-Rosell RC. Desafíos de la educación virtual en Odontología en tiempos de pandemia COVID-19. Rev Cubana Estomatol. 2020; 57 (3): e3341.

7. Tiol-Carrillo A. Aplicación de las tecnologías en la educación en odontología durante la pandemia por COVID-19. Rev ADM. 2021; 78 (3): 155-161.

8. Morgan $\mathrm{CH}$, O'Reilly M. Assessing open and distance learners. London: Kogan Page; 2002.

9. Khalaf K, El-Kishawi M, Moufti MA, Al Kawas S. Introducing a comprehensive high-stake online exam to final-year dental students during the COVID-19 pandemic and evaluation of its effectiveness. Med Educ Online. 2020; 25 (1): 1826861.

10. Rodríguez LM. Manejo de herramientas didácticas virtuales exitosas para el proceso de formación en presencialidad remota en el área de la salud. Espiral, Revista de Docencia e Investigación. 2020; 10 (1 y2): 143-152.

11. Royal College of Surgeons. Guidance for the Remote Delivery of Dental Exams. 2021. Available in: https://www.rcseng.ac.uk/ education-and-exams/exams/dental-examination-online-delivery/

12. Sáenz-Rangel S, Pérez-Quintero MT, Rodríguez-Luis OE, AraizaVázquez MJ. Creación de exámenes en línea como evaluación en entornos virtuales. Universidad Autónoma de Nuevo León, Facultad de Odontología [Internet]. 2018.

13. Zitzmann NU, Matthisson L, Ohla H, Joda T. Digital undergraduate education in dentistry: a systematic review. Int J Environ Res Public Health. 2020; 17 (9): 3269.

14. Reynolds PA, Rice S, Uddin M. Online learning in dentistry: the changes in undergraduate perceptions and attitudes over a four year period. Br Dent J. 2007; 203 (7): 419-423.

15. Sudarso S, Rahayu AP, Purnamasari CB, Fikriah I, Feri G. The impact of the online exam module on student anxiety during the COVID-19 pandemic. International Conference on Medical Education; 2021. 
Conflicto de intereses: Se respetó tanto el anonimato de encuestadores y encuestados con el fin de hacer un instrumento más libre y sin conflicto de intereses.

Aspectos éticos: Todos los participantes elegibles recibieron información a través de los encuestadores y antes de empezar a responder el cuestionario se les presentó a los participantes una descripción general del estudio general. Todos los participantes tuvieron la oportunidad de aclarar dudas antes, durante y después del estudio. No se ofreció ningún incentivo para participar.

Financiamiento: El financiamiento del proyecto fue por parte de los académicos involucrados.

Correspondencia:

Ricardo A Rivas Muñoz

E-mail:savir.zonum@gmail.com 